\title{
PENSAR LA EDUCACIÓN ARTÍSTICA EN EL CURRÍCULO NACIONAL: EVIDENCIAS, REFLEXIONES PARA IMAGINAR UN NUEVO ESCENARIO DE LA ASIGNATURA EN EL SALVADOR
}

Marta Eugenia Valle Contreras Universidad Dr. José Matías Delgado mevallec@ujmd.edu.sv

Recepción: 22 de agosto de 2016 Aceptación: 6 de septiembre de 2016 


\section{RESUMEN}

Tantas décadas de no atender el área artística en el país y, en consecuencia, en el currículo nacional -como reflejo de lo primero-, ha generado desafios de carácter estructural en la educación artística dentro del sistema educativo. Esto demanda pensar en una Estrategia Nacional para el Fomento de la Educación Artística desde el abordaje integral, sistémico y estratégico de la problemática para llegar al escenario deseado. Se resumen los desafios identificados en el estudio publicado, en 2011, por el Centro de Investigaciones en Ciencias y Humanidades (CICH) de la Universidad Dr. José Matías Delgado; se reportan avances y se ponen en contexto. Además se incluyen notas, a manera de preámbulo, a las puertas de entregar un nuevo estudio (desarrollado entre 2014-2016). Al final, se incorporan reflexiones para pensar en una Estrategia Nacional para el Fomento de la Educación Artística con el propósito de aportar a un abordaje integral de la problemática.

\section{Palabras Clave}

Educación artística - Estética - Cultura - Educación - Desarrollo social - Artes

- Ciencias humanas - Estrategia - Biocéntrico - Ciudadanía

\section{ABSTRACT}

As in decades the area of art education has not been taken care off in the national educative system. This has generated structural challenges for Art Education in the development of the national curriculum. This demands from the decision makers to think strategically to envisioned the changes and the desire future scenario. Here it is presented a summary of the findings from the research published, on 2011, by the Centro de Investigaciones en Ciencias y Humanidades (CICH), Universidad Dr. José Matías Delgado. As well, it is included notes from the new study (developed 2014-2016) to be published. At the end there are reflections to think about a National Strategy for the Development of Art Education in El Salvador proposing an integral and systemic approach to this national challenge.

\section{Keywords}

Artistic education - Esthetic - Culture - Education - Social development - Arts - Human Sciences - Strategy Biocentric - Citizenship 


\title{
PENSAR LA EDUCACIÓN ARTÍSTICA EN EL CURRÍCULO NACIONAL: EVIDENCIAS, REFLEXIONES PARA IMAGINAR UN NUEVO ESCENARIO DE LA ASIGNATURA EN EL SALVADOR
}

\author{
Marta Eugenia Valle Contreras \\ Universidad Dr. José Matías Delgado \\ mevallec@ujmd.edu.sv
}

\section{Introducción}

En 2011 se publicó La Educación Artística en la Enseñanza Básica en El Salva$d o r^{1}$, editado por el Centro de Investigaciones en Ciencias y Humanidades $(\mathrm{CICH})$ de la Universidad Dr. José Matías Delgado. Uno de los primeros productos del Programa de Investigaciones para el Fomento de la Educación Artística que se desarrolla en este centro de investigaciones desde 2008. Aquí se incluye un resumen de los aspectos que se valoraron en el diagnóstico de 2011 sobre esta área educativa en el país. De ese año a la fecha, hay una serie de avances y se mantienen muchos desafios; por lo tanto, a manera de preámbulo y a las puertas de entregar un nuevo estudio (desarrollado entre 2014 y 2016), este artículo encuadra las dimensiones que se valoraron en 2011 desde los actuales entornos.

\footnotetext{
${ }^{1}$ Marta Eugenia Valle Contreras, La Educación Artística en la Enseñanza Básica en El Salvador (Antiguo Cuscatlán: Centro de Investigaciones en Ciencias y Humanidades, CICH. Universidad Dr. José Matías Delgado).
} 
En este sentido, recoge notas del nuevo estudio a publicar por el CICH, Universidad Dr. José Matías Delgado, para repensar el papel de la Educación Artística en el currículo nacional. De la misma forma, se tocan temas relacionados como la formación pedagógica en esta área educativa y el papel del MINED en el desarrollo de la asignatura en los centros escolares del sector público; así como también otros aspectos de carácter estructural que afectan la calidad de la Educación Artística en la enseñanza básica, en el sistema educativo salvadoreño. Al final, se incorporan reflexiones para pensar en una Estrategia Nacional para el Fomento de la Educación Artística con el propósito de aportar a un abordaje integral, sistémico y estratégico de la problemática; de manera que sea considerado por la población y los tomadores de decisiones.

\section{Por qué pensar la educación artística en el currículo nacional}

Martha Nussbaum², filósofa norteamericana, es elocuente al respecto en su discurso al recibir el Doctorado Honoris Causa por parte de la Universidad de Antioquia, en diciembre de 2015. En este hace referencia a una crisis mundial de la educación, a la cual califica como una crisis de proporciones masivas y de grave importancia mundial... potencialmente devastadora para el futuro de la democracia en el mundo. Nussbaum continúa:

«Las humanidades y las artes están siendo eliminadas, tanto en la educación primaria/secundaria como en la técnica/universitaria, en prácticamente todas las naciones del mundo, vistas por los responsables políticos como adornos inútiles, en momentos en que las naciones deben cortar todas las cosas inútiles con el fin de mantener su competitividad en el mercado global, estas están perdiendo rápidamente su lugar en los planes de estudio y también en las mentes y corazones de padres y niños. De hecho, lo que

\footnotetext{
${ }^{2}$ Marta Nussbaum, Discurso al recibir el Doctorado Honoris Causa. Antioquia. 10 de Diciembre del 2015. Consultado en http://www.elheraldo.co/educacion/el-duro-discursode-martha-nussbaum-sobre-el-futuro-de-la-educacion-mundial-233416. Última consulta: agosto 18, 2016.
} 
podríamos llamar aspectos humanísticos de la ciencia y las ciencias sociales -el aspecto creativo imaginativo y el aspecto del pensamiento crítico riguroso- también están perdiendo terreno, debido a que las naciones prefieren perseguir beneficios a corto plazo cultivando habilidades útiles y altamente aplicables, adaptadas a fines lucrativos».

En su discurso, Martha Nussbaum valora además el equilibrio que debe haber en los sistemas, entre las áreas de conocimiento, para mantener saludable el currículo (si aspiramos a una sociedad saludable) en los siguientes términos:

«El afán de lucro sugiere a los políticos más preocupados que la ciencia y la tecnología son de crucial importancia para la salud futura de sus naciones. No debe haber ninguna objeción a una buena educación científica y técnica, y no sugiero que las naciones dejen de tratar de mejorar en este sentido. Mi preocupación es que otras habilidades, igualmente cruciales, están en riesgo de perderse en el frenesí competitivo. Habilidades cruciales para la salud interna de cualquier democracia, y para la creación de una cultura mundial decente, capaz de abordar de manera constructiva los problemas más apremiantes del mundo. Estas habilidades están asociadas con las humanidades y las artes: la capacidad de pensar de manera crítica; la capacidad de trascender las lealtades locales y acercarse a los problemas mundiales como un ciudadano del mundo y la capacidad de imaginar comprensivamente la situación del otro".

Por su parte, Teresa Torres Eça (Portugal) ${ }^{3}$ caracteriza la educación artística hoy, dejando claros los desafíos para la educación artística en el currículo nacional salvadoreño. La educadora e investigadora valoriza la Educación Artística como eje aglutinador de asignaturas y como impulsador de la diversidad cultural y la conciencia ambiental, al igual que como facilitador de debate en torno a aspectos de valores y de ciudadanía. Dimensiona a las artes para la recreación, enfatiza que están asociadas a las necesidades psicoló-

\footnotetext{
${ }^{3}$ Teresa Torres Eça. «A Educaçao artística e as prioridades educativas do inicio do seculo XXI». Revista Ibero Americana de Educaçao. N. ${ }^{\circ} 52,2010$.
} 
gicas básicas, siendo la recreación necesaria a todos los seres humanos ${ }^{4}$. Pero también Torres Eça, señala que las artes redireccionadas hacia la educación estética, para el desarrollo humano, se orientan hacia un concepto de educación estética y cultural de ciudadanía, que busca una formación integral de las personas para que se reconozcan como seres sociales e históricos, capaces de crear y re-crear su propia existencia; conceptos también consistente con Barbosa, A., (2007) ${ }^{5}$ y Aguirre, I., (2005).

Para Torres Eça, este concepto de educación artística implica que por medio de sus áreas y modos propios de pensar y hacer arte la educación artística presenta un modelo pedagógico, el cual ha demostrado el desarrollo de capacidades y destrezas, tales como aprender a aprender y aprender a ser, desarrollando capacidades de análisis crítico, de resolución de problemas y curiosidad por los fenómenos. Por lo tanto, sigue la autora, la Educación Artística no es un lujo. Desde este enfoque, la práctica de las artes permite que la niñez y juventud adquieran conciencia del mundo donde viven y tengan medios para resistir e intervenir en su comunidad. No se trata de una práctica solo tecnicista o instrumental, más bien se trata de una práctica artística milenaria que los artistas y educadores retoman de nuevo: la práctica social del artista activista, facilitador de procesos psicológicos y sociales, de construcción de significados y de enriquecimiento cultural.

Según Imanol Aguirre, el objeto del arte es concentrar experiencias dispersas de una manera estética y, por ello, el de la educación artística debe ser generar sensibilidad para hacer uso de dichas experiencias, llevándolas a las experiencias personales y así entrecruzarlas con la de los otros. Esta función de la Educación Artística para Aguirre va a brindar también

«(...) Amplia información sobre los léxicos ajenos, de forma que su familiaridad con ellos nos ponga en disposición de solidarizarnos con las causas justas. Conocer y comprender el léxico del otro es la vía para 'identificar-

\footnotetext{
${ }^{4}$ Leontiev, 2000, p.43. Citado en Torres E., Teresa, 2010.

${ }^{5}$ Ana Mae Barbosa, «Mudanças na Arte». São Paulo:Cortez, 2007. Obtenido de Texsituras: https://texsituras.files.wordpress.com/2010/04/anamae.pdf Última consulta: 18 de agosto de 2016.
} 
nos' con el otro y ‘hacerlo de los nuestros'. La educación artística es idónea en ese sentido». ${ }^{6}$

Por ello, Aguirre propone el cambio de nombre de la asignatura en el currículo: de Educación Artística a Educación Estética porque este último -bajo una acepción contemporánea de la estética- no aspira ya exclusivamente a formar el gusto y la sensibilidad estética, ni tampoco se limita al enjuiciamiento de las producciones artísticas, sino se ve como un tipo básico de $\operatorname{cognición}^{7}$ que proporciona una perspectiva de enfoque que suministra categorizaciones útiles para extraer significado de las prácticas humanas y para generar conocimiento. ${ }^{8}$

Esto, sin dejar de lado la producción artística (sigue Aguirre) porque se trata de una materia de algo más que de estudio y análisis. Así conlleva la formación «en y para» las experiencias estéticas, para lo cual debe reorientarse hacia la formación crítica y el enjuiciamiento, como hacia la elaboración de artefactos estéticos; es decir, productos que resultan de aplicar la mirada especial de la estética sobre la realidad.

Desde esta perspectiva estética, cuyas dimensiones explica Aguirre, cabe mencionar el papel que brinda la Educación Artística (estética y cultural) para facilitar experiencias significativas de aprendizaje que contribuyan a asimilar con pensamiento crítico las innovaciones tecno científicas.

Esto es porque, según Medina, las innovaciones tecno científicas -al igual que las demás realizaciones culturales ${ }^{9}$ - configuran sistemas culturales constituidos por el colectivo de los portadores de la cultura particular junto con las prácticas específicas de su identidad cultural. Esto se traduce en los procedimientos y formas de acción e interacción reproducibles, transmisibles y generalizables y, por tanto, generadores de cultura; por ello, los sistemas culturales y los procesos que llevan a las innovaciones en general, y en

\footnotetext{
${ }^{6}$ Imanol Aguirre, Teorías y prácticas en educación artística: ideas para una revisión pragmatista de la experiencia estética. (Barcelona: Editorial Octaedro, S.L.), 329.

${ }^{7}$ Parsons, 1987. Citado en Aguirre (2005, p.338).

${ }^{8}$ Imanol Aguirre, Teorías y prácticas en educación artística: ideas para una revisión pragmatista de la experiencia estética. (Barcelona: Editorial Octaedro, S.L.), 338.

${ }^{9}$ Manuel Medina, Ciencia, Tecnología, Sociedad y Cultura en el cambio de siglo: Ciencia y tecnología e innovación como sistemas culturales. (Madrid: Biblioteca Nueva,), 85.
} 
particular tecno científicas, así como estas mismas son también sujeto de la Educación Artística (estética y cultural) y han sido sujeto de interacciones que han resultado, históricamente, en influencias mutuas entre ciencia, artes y tecnología.

Medina sostiene que esto es así, ya que todo colectivo-práctica cultural viene mediado artefactualmente, interpretado de manera simbólica, articulado socialmente y situado ambientalmente por su correspondiente entorno, constituido por el legado cultural que, a su vez, conforma de manera estructural la práctica del sistema determinado.

Al respecto, explica Medina, dicho legado cultural comprende el entorno material o conjunto de artefactos, técnicas y construcciones materiales; el entorno simbólico, de las conceptualizaciones, representaciones, interpretaciones, legitimaciones y valores; el socio entorno de las instituciones y formas de organización e interacción comunitarias, sociales, económicas, jurídicas y políticas, las reglas, roles, normas fines, etc. y el bioentorno o comunidades de seres vivos y medio biótico implicados.

Finalmente, el enfoque educativo biocéntrico ${ }^{10}$-creado por Rolando Toro-, es una propuesta integradora de las diferentes dimensiones de la existencia, principalmente de los aspectos culturales y biológicos, prevaleciendo como eje referencial todos los procesos de la vida que colaboran en el mantenimiento y la expansión de la misma. En este sentido, el enfoque educativo biocéntrico retoma la vinculación de la elaboración estética de las formas y significados mediados por el universo creativo y sus iniciativas con el acto de vivir; lo que contribuye a la expansión de la conciencia ecológica, artística, ciudadana, planetaria, social, cultural, del arte originario, etc.

En conclusión, las experiencias estéticas en la Educación Artística (de acuerdo a Aguirre, 2005; Torres Eça, 2010), como se ha perfilado en este texto, que conllevan las prácticas artísticas (analizadas por Torres Eça, 2010) brindan al alumnado la oportunidad de vivenciar en la asignatura de Educación Artística, del sistema educativo nacional, procesos psicológicos y sociales de construcción de significados y de enriquecimiento cultural.

\footnotetext{
${ }^{10}$ Rolando Toro, Creador de la Biodanza y el Enfoque educativo Biocéntrico. Para ampliar ver: http://www.biodanza.org/es/biodanza. Sobre el enfoque educativo biocéntrico se cita el texto inédito en preparación de dos de sus colaboradores, Osvaldo Marcón y Nélida Pérez, que han sistematizado sobre este enfoque.
} 
La sociedad salvadoreña puede potenciar esta valiosa oportunidad, si dicha asignatura es mediada desde un enfoque que hace posible los alcances de la Educación Artística, en los términos que se ha esbozado aquí por los autores antes citados. De lo contrario, el sistema educativo cae en la práctica artística solamente de carácter tecnicista o instrumental.

El enfoque contemporáneo de la Educación Artística ha elaborado y sistematizado estas potencialidades, habría que pensar la asignatura en El Salvador desde esta perspectiva; en todo caso, hay consensos internacionales sobre el sentido que debe tener la educación artística en el currículo, con variaciones propias de cada región consistentes con lo que aquí se plantea. Dichos ajustes obedecen a aspectos históricos y socioculturales; sin embargo, predomina el sentido contemporáneo en los términos que representan los autores citados.

Potenciar la asignatura de Educación Artística en el sistema educativo salvadoreño es urgente, dada la realidad del contexto nacional y la presión que esta genera a la ciudadanía; en particular sobre la niñez y la juventud. Hasta aquí se ha expuesto el potencial de esta área del currículo para el crecimiento personal y el desarrollo cultural que lleve al desarrollo integral de los salvadoreños y del país en general; esto depende de factores estructurales que se reseñan a continuación.

\section{Aspectos metodológicos}

Se resumen los aspectos metodológicos que llevaron a las principales conclusiones -aquí presentadas- del estudio realizado en 2011 sobre el estado de la Educación Artística en la enseñanza básica en el país. En estas conclusiones, se recoge la visión de alumnos, docentes, personeros del MINED, formadores de maestros, empresarios, intelectuales, gestores culturales y artistas sobre el desarrollo de la asignatura de Educación Artística y las artes en general en El Salvador. 
Del mismo modo, esta investigación cualitativa exploratoria ${ }^{11}$ incluye una amplia investigación documental de carácter histórico y de literatura especializada en Educación Artística contemporánea, como área de conocimiento. ${ }^{12}$ En las conclusiones presentadas en este artículo se retoma la dimensión histórica y se efectúan conclusiones sobre los hallazgos.

En la primera fase se entrevistaron 43 personas involucradas en el campo de la educación, educación artística, gestión cultural, empresarial y gubernamental del país. Se utilizó un muestreo por conveniencia Delphi (entrevista con expertos) y se integró la muestra por medio de la técnica Bola de nieve. De acuerdo a esta técnica, una persona recomienda a otra hasta que la muestra se satura y los informantes empiezan a repetir sus argumentos de forma recurrente.

Por otro lado, se encuestaron 20 maestros de un grupo que incluyó docentes de distintas zonas del país, a quienes se les solicitó (por convenirle más a ellos) que contestaran por escrito la misma guía, con alguna variación, utilizada con el grupo entrevistado personalmente.

En la segunda fase, se tomó una muestra de 20 escuelas del área de influencia de la Universidad Dr. José Matías Delgado (departamento de la Libertad). El número de escuelas se determinó de acuerdo a los recursos (humanos, financieros, equipo, etc.) y tiempo disponibles para el proyecto. La muestra de centros escolares fue seleccionada al alzar a partir de la base de datos del MINED, utilizando una tabla de números aleatoria. En estas escuelas se entrevistó a directores, docentes de enseñanza básica y a un estudiante de cada uno de los grados de segundo ciclo.

Con las personas que colaboraron en las dos fases de la investigación, incluyendo a las entrevistadas en los centros escolares, se efectuaron

\footnotetext{
${ }^{11}$ Víctor Navas, La Investigación Social. (San Salvador: Centro de Investigaciones en Ciencias y Humanidades (CICH-UJMD), Universidad Dr. José Matías Delgado. 2012, 21.

${ }^{12}$ Para ampliar sobre los temas tratados en este artículo en el libro La Educación Artística en la Enseñanza Básica en El Salvador (Valle Contreras, 2011) el lector encontrará en la Primera Parte un amplio recorrido sobre las artes en el currículo nacional, a partir del período Colonial Tardío hasta 2010, año cuando se cerró el estudio de 2011. De igual manera, encontrará el desarrollo de los conceptos y tendencias contemporáneas en educación artística y aspectos metodológicos de dicha investigación.
} 
entrevistas cualitativas ${ }^{13}$ con preguntas descriptivas ${ }^{14}$ y se utilizó un cuestionario como guía o entrevista por pautas. Aquí se presentan algunos de los segmentos citados en el estudio de 2011.

Entre todas las entrevistas realizadas para dicho estudio, este segmento transcrito resulta emblemático por contener elementos retomados aquí para vislumbrar los avances logrados y también para detectar los desafios en esta área en el currículo nacional, desde ese año a la fecha.

La respuesta de un maestro a la pregunta sobre los requisitos solicitados para aplicar a la plaza de docente de Educación Artística en una institución del sector privado fue:

«(...) Solo me pidieron currículum de mi experiencia laboral e información profesional. Me comentaron que no había mucha exigencia, en el sentido que esas asignaturas de arte no estaban formalizadas en el Ministerio, pues en las escuelas no las dan, que no había una escuela de formación para profesores de arte; entonces pues, que no había mucho requerimiento, pero sí (era necesario) que tuviera una experiencia».

Y sobre su estatus laboral ante el MINED, manifestó: «Hasta antes del año pasado (2007) no lo exigieron», refiriéndose al título de profesor.

Los elementos recuperados en la investigación de 2011 son:

1. Conocer la ubicación geográfica del centro escolar, infraestructura, aspectos socioeconómicos y culturales de la comunidad donde se encuentra.

2. El concepto de la asignatura de Educación Artística manejado por los docentes.

3. Evidencias del desarrollo de la materia en cada una de las escuelas visitadas: descripción de los métodos y las técnicas pedagógicas (la didáctica). Los recursos con los que cuentan para

\footnotetext{
${ }^{13}$ Honorio Velázco, y Ángel Díaz de Rada, La lógica de la investigación etnográfica. Un modelo de trabajo para etnógrafos de la escuela: El Trabajo de Campo (Madrid:Trotta), 2007.

${ }^{14}$ Rafael Flórez Ochoa, y Alonso Tobón Restrepo. Investigación educativa y pedagógica. (Bogotá: McGraw Hill Interamericana, S.A.), 1997. 54.
} 
el desarrollo de la asignatura. Las teorías pedagógicas que subyacen en la base del trabajo docente en esta área educativa. La organización de los grados (integrados o no), las condiciones físicas de las aulas.

4. Identificar y documentar los aspectos referentes a la formación de los docentes en el área de Educación Artística y su efecto en el desarrollo de la misma.

5. Establecer el papel del Ministerio de Educación en el desarrollo de la asignatura de Educación Artística en los centros escolares públicos visitados.

\section{Temas clave}

Los temas clave y emergentes que surgen al responder las preguntas de sistematización son:

1. La calidad de la Educación Artística en la enseñanza básica en El Salvador.

2. La funcionalidad de esta asignatura como medio para la educación cultural en general y, en particular, para la aproximación informada y activa de la niñez a los hechos artísticos.

3. El potencial de la Educación Artística para identificar y promover jóvenes talento en las diferentes ramas artísticas, en etapas tempranas de su desarrollo biosicosocial.

4. La funcionalidad de la asignatura de Educación Artística como medio para la formación humana:

-Su contribución específica en la formación integral y en el desarrollo creativo y socio afectivo de los educandos.

-Su contribución para acercar a los educandos al mundo de las artes, con el fin de que las disfruten en plenitud como público o creadores.

-Su contribución en la formación de ciudadanía, en la enseñanza básica, y como apoyo fundamental en los procesos de formación de identidad cultural, memoria histórica, 
participación ciudadana, valores solidarios, respeto a los derechos humanos, a la multiculturalidad, entre otros.

5. Las artes como apoyo al desarrollo de las otras asignaturas del currículo (aspecto multidisciplinario).

6. El papel del Ministerio de Educación en el desarrollo de la calidad de la asignatura de Educación Artística en el país.

7. La formación docente de los maestros de Educación Artística en el sistema educativo salvadoreño.

\section{Temas emergentes relacionados}

1. Las artes como opción para mejorar la calidad del tiempo de ocio de los salvadoreños.

2. El apoyo social que se le da al desarrollo de las artes en el currículo nacional.

3. La formación especializada en Educación Artística en El Salvador.

4. La Educación Artística en el país, en relación a su desarrollo en la región centroamericana, latinoamericana y el mundo.

A estos temas hay que agregar el amplio interés que han suscitado las artes como medios para la creación y el empoderamiento tecnológico, así como el papel importante para la creación de marcos mentales para la innovación - que si bien no pasa necesariamente por el dominio y práctica artística- ser sensibles y alfabetizados, estética y culturalmente, son rasgos importantes de los perfiles innovadores a lo largo de la historia.

Evidencias recopiladas (2008-2010)

\section{La calidad de la enseñanza y desarrollo de la asignatura de Educación Artística}

Lo manifestado por los expertos apunta a que es complicado impartir la materia de Educación Artística en los centros escolares del sector público del país. 
Algunos maestros consultados manifiestan que no imparten la asignatura por falta de formación docente en el área de Educación Artística, al igual que por la imposibilidad de tener recursos como equipo (instrumentos y CD player para Educación Musical, por ejemplo) y materiales (para Dibujo y Pintura, entre otros). Algunos de los centros escolares en los que se argumenta lo anterior son poco accesibles y están ubicados en comunidades muy pobres.

La falta de recursos para impartir la materia no es la única razón por lo que esta asignatura no se desarrolla. Tal aspecto -junto con la sensación que tienen los docentes de no dominar la materia- predispone para que ellos decidan no impartirla. Estos escenarios, en conjunto, son los que proporcionan argumentos a los directores y docentes para no desarrollar la Educación Artística de manera regular, tal como se trabajan otras asignaturas.

Sin embargo, en algunos de estos centros escolares - al preguntar a los maestros por el aporte potencial de las artes en la formación de la niñezestos reconocen que las artes son importantes. Así lo manifiesta el director de uno de los centros en condiciones precarias:

«Los niños vienen con estrés emocional crítico porque los padres no tienen cómo subsistir; los padres se vuelven alcohólicos, etc. (sic). Sería maravilloso que nos apoyaran en ese sentido (formación docente y recursos para la Educación Artística); con base en las pruebas cognitivas, los niños están mal».

Otros directores manifestaron que no desarrollaban la asignatura porque «(...) Se le da prioridad a las (materias) básicas. Se refieren específicamente a Matemáticas, Estudios Sociales, Ciencias, Lenguaje y Salud y Medio Ambiente». De manera recurrente, los maestros entrevistados expresaron que refuerzan las asignaturas de Matemática, Lenguaje e Inglés en el tiempo asignado a la Educación Artística. Argumentan, por ejemplo, que:

«Se le da prioridad a Inglés porque no tenemos maestro de Educación Artística, pero sí de Inglés. Además estamos enfocados en Lenguaje y Matemáticas porque las están evaluando... En esta realidad la gente está sobre- 
viviendo y lo artístico es más para un nivel más especial (un nivel socioeconómico superior del alumnado)».

En otros centros escolares los maestros aseguran que:

- «Como decisión pedagógica del centro escolar no se imparte Artística sino Inglés, de $6^{\circ}$ a $9^{\circ}$ grado para que (los alumnos) ya tengan una base».

- «No la estoy impartiendo (sic) (Educación Artística) porque el tiempo no alcanza, le doy prioridad a las (materias) básicas».

- «Utilizo el programa antiguo (2004). El tiempo es muy corto, les doy una hora a la semana; las otras tres horas las utilizo para reforzar Matemáticas o Lenguaje porque les doy prioridad».

Estas citas revelan, por un lado, que los docentes entienden la existencia de asignaturas (del currículo nacional) y las perciben como básicas, mientras que otras no lo son. Entre estas últimas se encuentra la Educación Artística. Igualmente, evidencia la percepción que tienen los maestros sobre su propio desempeño y los logros de los estudiantes, en dicha asignatura, no están siendo "evaluados» por el MINED. Por tanto, dejar de desarrollar la Educación Artística - para extender las horas dedicadas a las materias a evaluar por el Ministerio de Educación en la Paesita, ${ }^{15}$ - es una de las formas en que estos docentes priorizan las que consideran «básicas» en el currículo nacional, y por cuyos logros sí serán evaluados tanto ellos como sus alumnos.

De igual manera, se evidencia la creencia de algunos maestros y directores: que la Educación Artística es un «lujo» (no un derecho humano y cultural. UNESCO, 1997, 1998, 2001, 2006, 2009), que se trata de una disciplina con la cual la niñez pobre no debe «perder el tiempo», dada su carente condición socieconómica.

\footnotetext{
${ }^{15}$ Se refiere a las pruebas de logros conocidas como Paesitas, realizadas por el MINED a niños de tercero, sexto y noveno grados; se aplican cada tres años para medir el progreso educativo de los educandos en primer ciclo, en las asignaturas de Lenguaje y Matemáticas. A partir de 2008,0 se incluyen las asignaturas de inglés e informática en esta prueba. «MINED incluirá Informática e Inglés en la PAES», La Prensa Gráfica, mayo 18, 2008, acceso marzo 10, 2010, en http://archive.laprensa.com.sv/20080518/nacion/1061253.asp
} 
Así, la Educación Artística no es considerada un campo válido como área de conocimiento, ni como potencial fuente para el desempeño laboral capaz de generar ingresos. En estos centros escolares no se le valora como conocimiento útil para el desarrollo laboral futuro, de tal manera que se descarta de la jornada escolar regular y prevalecen otras asignaturas. Otros docentes entrevistados creen que la preparación de presentaciones de carácter artístico, con algunos grupos de estudiantes para las fechas festivas (Día de la Familia) o conmemorativas (15 de Septiembre), es suficiente para suplir la ausencia, en la práctica, de la asignatura de Educación Artística.

\section{El enfoque de la asignatura de Educación Artística}

El currículo nacional en la enseñanza básica en el país es prescrito. Las entrevistas a los docentes reflejaron que predomina el uso de los programas de 2004; incluso algunos de ellos no los utilizan. Al momento de las entrevistas, apenas algunos profesores afirmaron adquirir por Internet los programas de 2009 , bajo el enfoque por competencias. Como se ha mencionado, no se incluyó Educación Artística en los paquetes de material bibliográfico de apoyo a la edición de estos, como tampoco capacitaciones en esa área educativa, por lo que aun cuando algunos educadores tienen los programas, no han podido replantear su práctica docente en esa materia.

Esto tiene un impacto en el enfoque brindado a la asignatura de Educación Artística en el país, debido a que depende en gran medida de la formación docente específica en esta rama educativa o de la ausencia de esta. Los formadores de docentes entrevistados en $2009^{16}$ coinciden en que, en la enseñanza básica, predomina la tendencia de los maestros a enfocar la asignatura de Educación Artística como manualidades.

Ante la falta de formación para desarrollar el programa prescrito de tal asignatura, los docentes entrevistados que sí trabajan con Educación Artística optan por:

\footnotetext{
${ }^{16}$ Alberto Escobar, y Antonio Bonilla, Universidad Pedagógica; Álvaro Cuestas, Universidad de El Salvador. También asesores pedagógicos y gerentes del MINED, maestros entrevistados y Verónica Miranda (Artes Visuales).
} 
- Dedicarse a desarrollar manualidades (establecidas por ellos o tomadas de los programas de 2004) y combinarlas con Dibujo. ${ }^{17}$ Haciendo a un lado la Educación Musical y Teatro, contempladas en los programas de 2004 y 2009; o, en su defecto, tomar algunos contenidos de estas últimas y no abordar las artes plásticas.

- Desarrollar algunos de los contenidos del Programa de Educación Artística de 2009, sobre todo los de Artes Visuales, relegando los de Educación Musical y Teatro.

\section{Recursos}

El enfoque de la asignatura, además de relacionarse con la formación docente, es un aspecto vinculado al acceso a materiales e infraestructura. Esto tiene relación importante con las condiciones precarias en que trabajan algunas escuelas que si bien no es absolutamente determinante, constituye una realidad que afecta la calidad de la Educación Artística y la manera cómo la abordan los docentes.

Las siguientes citas son elocuentes:

«Recibimos los programas de primer ciclo y tenemos capacitaciones en evaluación. Trabajamos con el programa de 2004. La compañera que lo da (sic) (educación artística)...usa los programas antiguos porque no tienen material para dar los nuevos (2009, segundo ciclo)».

Refiriéndose a la asignatura de Educación Artística, algunas docentes manifiestan:

«Manualidades no se les da por la falta de recursos. Se les pide papelería, pero por la situación económica no la traen. Así fue el año pasado».

\footnotetext{
${ }^{17}$ Predomina mucho el dibujo en cuaderno cuadriculado de figuras geométricas en los cuales los alumnos pintan los cuadros de diferentes colores hasta obtener una figura. Luego, es bastante generalizado el dibujo libre, como también copiar dibujos de la pizarra, calcomanías o de libros de historietas.
} 
En esta escuela no se imparte la asignatura de Educación Artística; lo expresado por estas docentes refleja que, para ellas, las manualidades equivalen a ofrecer esta disciplina.

«El problema es que, por la comunidad, los alumnos no tienen materiales para hacer las actividades; por ejemplo, manualidades. Sus padres venden un poquito de su cultivo fuera del cantón y el resto es para consumo propio. Por eso optamos por el trabajo del Dibujo y habilidades de diseño de cuadrículas (geométricos pintados en cuaderno cuadriculado). Para dar Canto no tenemos el aparato para escuchar la música. Me interesa trabajar con música tradicional, los niños en su casa oyen música grupera.

Hacemos Teatro en Lenguaje (la asignatura), Danza no. Hay un comité cultural de apoyo de grado para la semana cultural que trabaja fuera del horario de clases (preparan presentaciones para estos actos). Tenemos lápices de grafito y algunos de color. Compramos las cajas para uso común en algunos años, ahora la Alcaldía dona algunas cajas. No tenemos acceso a materiales de modelado, solo parvularia. Sí hay computadoras con Windows, se le está dando Computación a Tercer Ciclo. No aparece como asignatura, por lo cual son clases extra y eso no se permite.

La Música no se desarrolla, nos inclinamos por manualidades debido a la pobreza de los estudiantes. La música folclórica se desarrolla con un CD player de baterías.

En Danza se preparan (presentaciones) para la semana cívica, hay un grupo de niñas que se integraron hace unos cuatro años. No cumplimos el programa como es porque es diferente, porque los niños no tienen los recursos, no los traen (el material) y no hay energía en todo el cantón y por la calle (mala), no tienen transporte público. Con la luz y la energía se podrían hacer (formar) alumnos más competentes. Por eso nos inclinamos más a las manualidades hechas con papel, a la elaboración de piñatas: payasitos de papel, portalápices de cartón corrugado o los centros de papel higiénico con papel retorcido.

No se les puede pedir materiales como el 'fomi' (espuma delgada), papel o lápices de color por la pobreza de los padres.

Los estudiantes se costean los materiales. El año pasado trabajé con barro, luego se puso a secar al sol y se pintó en frío con témperas. Trabajé flores 
de papel. Me gustaría tener materiales que los jóvenes no se pueden costear como acrílico y óleo. Me gustaría que hubiera un CD con libro o video que pudiera verse y escucharse, a la vez, sobre Educación Artística».

\section{Contenidos que se abordan en los centros escolares que imparten la asignatura}

Los centros escolares visitados se encuentran en zonas urbanas y rurales. Algunos son grandes y están relativamente equipados; otros son pequeños y se hallan en condiciones precarias. Estos últimos predominan en las zonas rurales. Tanto en unos como en otros se hizo énfasis en la dificil situación económica de los padres de familia y en la dificultad para proveer, por tanto, de materiales para el trabajo.

Los maestros que imparten la Educación Artística en los centros escolares (rurales o urbanos) actúan con entusiasmo en el desarrollo de la misma. Algunos aprovechan la preparación artística que recibieron fuera de sus programas de formación docente; otros expresan que tuvieron muy buen maestro de Educación Artística durante el semestre que cursaron la asignatura, mientras estudiaban en la universidad. Algunos tratan de hacer lo que entienden de los Programas de 2004 o 2009 y otros buscan revistas y toman cursos de manualidades.

Muy pocos docentes han podido conseguir guías para la Educación Artística y, en alguna medida, las siguen o brindan la asignatura porque son bachilleres en artes. Entre los entrevistados predomina la visión que la Educación Artística es importante en el currículo por las siguientes razones: en primer lugar, desarrolla la motricidad fina. En segundo lugar, sirve como apoyo a las otras asignaturas; además ayuda a los educandos en su autoestima y también a expresarse mejor; estimula la creatividad y, por último, entretiene y relaja.

En este marco, los contenidos que desarrollan son actividades muy puntuales donde prevalece el dibujo en un cuaderno cuadriculado, de figuras geométricas simétricas, que son trazadas por los educandos, contando los cuadros y coloreando secciones hasta completar la figura utilizando toda la página. El dibujo libre. Copia de dibujos de la pizarra, de libros de cuentos e historietas y de los paquines o de series de dibujos animados. 
A la par del dibujo están las manualidades. En algunos centros escolares dedican todo el año a desarrollar proyectos con diferentes materiales; en algunos casos con productos reciclados. En la asignatura de Lenguaje, leen un cuento o poema y algunas veces lo ilustran. En Música predomina el canto de canciones con mensaje, la elaboración de sonajas y el uso de claves.

Al no contar con profesores de música en cada centro escolar, el programa prescrito vigente (2009) está diseñado de manera que los docentes puedan mediarlo con un mínimo básico disciplinar, obedeciendo al objetivo de desarrollar procesos educativos en Música que creen ambientes y experiencias significativas de aprendizaje que comprenden la percepción, expresión vocal e instrumental, el lenguaje y apreciación de la música en el entorno urbano, natural, doméstico, artístico y cultural; así como en la audición musical de distintos géneros, épocas y estilos. ${ }^{18}$

Las evidencias muestran que, en los programas de profesorado de educación básica, los docentes no desarrollan las competencias para impartir estos contenidos del programa de la asignatura de Educación Artística. Por otra parte, cuando un maestro posee formación para tocar un instrumento, los centros escolares no cuentan con los instrumentos para uso de los alumnos. Por ejemplo, durante las entrevistas, un docente que toca guitarra dijo que no enseña a los alumnos a tocarla porque estos no las tienen.

Los maestros solicitan a sus alumnos que en la clase de Lenguaje ensayen dramatizaciones de poemas, cuentos o fábulas. Asimismo, en la clase de Ciencias realizan algunas dramatizaciones. En otros casos, elaboran títeres y pequeños montajes teatrales.

\section{El papel del Ministerio de Educación en el desarrollo de la asignatura educación artística, infraestructura y formación docente}

Las condiciones de algunas escuelas visitadas son precarias. Por ejemplo, en uno de los centros escolares visitados, los maestros solicitaron 50 juegos de pupitres y sillas individuales para los estudiantes de educación básica.Ya no

\footnotetext{
${ }^{18}$ Ministerio de Educación, Programa de Estudios de Cuarto Grado (San Salvador: Ministerio de Educación), 2009.
} 
quieren los bancos para varios alumnos. Están saturados debido a que por el Programa Red Solidaria (Programa del gobierno saliente en 2009) les aumentó la matrícula. De la misma manera, solicitaron una persona para enseñar computación, pero no tienen local para impartir la materia, ni fondos para pagar los honorarios del maestro.

Otros profesores manifestaron lo inadecuado y dificil que resulta manejar las aulas integradas (aulas donde los niños, de hasta tres grados diferentes, reciben clases con un docente durante la misma jornada). En estos centros escolares las mesas y bancos están deteriorados, las áreas de tierra sin grama. Hay zonas amplias de tierra suelta que rodean las aulas, por tanto, los pasillos y las zonas interiores de las escuelas se llenan de polvo durante la época seca. La estructura tipo de las escuelas no tiene ventanas, solamente un enrejado. En algunos casos hay demasiados alumnos por aula, por lo que se dificulta circular entre las filas de pupitres u organizar el salón de otra forma.

Otras limitaciones que poseen las escuelas son la ausencia del servicio de agua y electricidad y que las calles de acceso son de tierra. Los niños de los cantones, quienes normalmente viajan a pie, se exponen a respirar (por kilómetros) las nubes de polvo suelto de estos caminos durante la época seca; mientras que durante la época lluviosa estos caminos se vuelven fangosos. Es necesario aclarar que no todos los centros visitados presentan estas condiciones.

Entre los docentes entrevistados existe la percepción de que el Ministerio de Educación no apoya el desarrollo de la asignatura de Educación Artística. Como evidencia de esto se menciona: a) la falta de presupuesto para comprar materiales y equipo específico para la asignatura; b) ausencia de capacitaciones y material bibliográfico de apoyo al área de Educación Artística; c) poca supervisión sobre el desarrollo de la materia en los centros escolares, sus problemas y logros; d) la no asignación de especialistas. A todo esto se suma la precariedad antes mencionada. Estos se vuelven factores que obstaculizan el desarrollo escolar en general y, seriamente, el de la asignatura en cuestión.

$\mathrm{Al}$ respecto, se entrevistó a tres asesores pedagógicos del Ministerio de Educación. Ellos confirmaron que, históricamente, los personeros del MINED han enfatizado la evaluación de los logros educativos con base a 
cuatro asignaturas: Lenguaje, Matemáticas, Sociales y Ciencias. Que ha habido una desatención de la Educación Artística y quizá cierto temor de los docentes... «de parte de, no un 100 por ciento de ellos, pero sí de un buen porcentaje, de hecho no la planifican sino que ocupaban esas horas en otras asignaturas», expresó un asesor.

Según los asesores, los maestros consideran que se necesita un especialista que imparta y domine el área específica de la Educación Artística. Para mejorar esta situación, estos funcionarios recuerdan que se impartieron capacitaciones en esa área durante 2003; además mencionan que, como asesores, orientan a los docentes para que planifiquen y desarrollen la asignatura.

A partir de los programas de 2008, el MINED ofreció lineamientos a los docentes en el sentido que su deber es atender la asignatura de la misma manera que las otras, incluso con igual ponderación en la evaluación.

Los asesores expresaron desconocer por qué se ha dado esta situación en el MINED. Uno de ellos manifestó que, cuando estudiaba profesorado, había docentes especialistas en Educación Estética en las escuelas y que ahora los maestros cuestionan la ausencia de estos en el actual sistema, por considerar que se trata de una vocación. Sin embargo, uno de los asesores, dijo orientar a los maestros asegurando

«(...) Que todos tenemos habilidades de cantar y dar la parte de (Artes) Plásticas y Artes Escénicas. Que en la formación docente esos son temas de trabajo. Si todos hemos pasado por la universidad, todos tenemos bases para desarrollarnos en este tema a la par de los niños, si es que no la hemos desarrollado».

A la pregunta sobre si es necesario nombrar especialistas en Educación Artística, respondieron que sí lo consideran necesario; pero que en el país no hay. Aunque se podría desarrollar como segunda especialidad. Uno de los asesores mencionó que conoce la experiencia de un centro escolar, del sector público, donde labora una maestra especialista en Educación Artística nombrada para el centro y quien imparte clases desde primero a noveno grado. 
«(...) Y a los niños les gusta la clase de artística porque es especialista (la docente). Les enseña mucho más de lo que el currículo pide. Así que los niños en esa materia se sienten bien. El Ministerio la ha nombrado en este departamento. Todavía hay ese tipo de nombramientos».

Este asesor pedagógico piensa que de esta manera se tendrían que incorporar los especialistas en el sistema.

Por ahora, los asesores declaran que tratan de ofrecer apoyo a los docentes de grado para que desarrollen la asignatura. Aunque no son especialistas en Educación Artística, ven la dificultad de los docentes para ofrecerla. Una de las asesoras considera que un 50 por ciento de los docentes en su distrito imparten la asignatura como pueden; mientras que el otro 50 por ciento no la está desarrollando.

Un personero de nivel gerencial del MINED, del Gobierno anterior (2004-2009), manifestó que

«(...) Estamos conscientes de que los docentes confunden la asignatura con manualidades. Por eso se entiende como algo más simple y menos trascendental en la formación de toda persona. Pero, tenemos muy claro la relevancia de la asignatura y la importancia que no desaparezca (del currículo) para desarrollar el pensamiento crítico (entre otras cosas), y que permita una participación ciudadana eficaz. Porque amplía la visión de mundo de los niños y su sensibilidad, su marco de referencia y sus capacidad expresiva, siendo una riqueza que abarca diferentes dimensiones del ser humano desde lo cognitivo, lo psicomotor, el tema de valores, porque el arte humaniza».

\section{Casos especiales}

En la publicación de 2011 (Valle Contreras, p. 220) se incluye el estudio de casos especiales encontrados. En estos centros están logrando potenciar la Educación Artística alineando personal docente de educación básica con el alumnado y la Dirección del Centro Escolar para maximizar los recursos y así explorar la oportunidad que significa la Educación Artística en el sentido descrito anteriormente. 
Uno de los casos es el del Centro Escolar «Mario Calvo Marroquín», de Izalco; en este centro se recupera la lengua Náhuat, se enseña de manera sistemática desde primer grado. La escuela cuenta con un grupo de proyección folclórica; su repertorio musical incluye canciones en el idioma que hablaron sus ancestros e interpretan la música de canciones sobre sus tradiciones, vida y costumbres. Esta iniciativa es apoyada por la Universidad Don Bosco y la Lic. Julia Ama de Chile (en 2011), directora del centro escolar, y es llevada a cabo por los profesores Atilio Enrique Carías Cruz y Raúl Alfonso Tula Ramos. Para ellos, las artes son una valiosa oportunidad de recuperar la lengua que forma parte de su cultura, al igual que una manera de compartir y dar continuidad a su patrimonio cultural.

En cuanto a la asignatura de Creatividad, en bachillerato, se da un caso especial en el Centro Escolar República del Japón, en Mejicanos. Para el profesor Luis Ramos, esta materia significa para los estudiantes la posibilidad de acercarse al mundo laboral. Él motiva a sus alumnos enseñándoles cierto tipo de manualidades para ganarse la vida. Los jóvenes empiezan pintando o elaboran tarjetas con la técnica de filigrana.

«(...) Al principio son apáticos, más los varones que las niñas. No traen habilidades básicas, les enseño por medio del desarrollo del trabajo. Los motivo con el tema de ganarse la vida. Hacemos macramé, repujado con latas de gaseosa; también se les pone una canción y luego la ilustran (en una serie de cuatro cuadros en secuencia), después llegan a desarrollar productos e, incluso, publicidad».

El segundo caso se trata del Complejo Educativo «José Simeón Cañas», cantón Julupe, Chiltiupán. Este refleja un alineamiento entre el alumnado de bachillerato con el profesorado de educación básica para potenciar la Educación Artística y Creatividad en ambos niveles del currículo.

En este Complejo se imparte la asignatura de Creatividad (en bachillerato) de una manera particular. Quien está a cargo es la directora del centro, Araceli Alas de Ramos. Ella ha desarrollado una modalidad en la cual realiza talleres de Educación Artística con los estudiantes de bachillerato; luego ellos replican lo aprendido en el nivel de educación básica. 
«Vienen fuera del horario de clases ordinario a hacer esto. La modalidad la desarrollé porque vi que los estudiantes de tercer año tenían tanta habilidad y frescura en sus ejercicios. Los jóvenes evalúan el trabajo realizado en la clase de Educación Artística con los estudiantes de básica y el maestro de grado los apoya.Ven Plástica: pintura de dedo y témpera; Música, Danza folklórica y Canto: canciones con mensaje. Se les da copia de la letra y la practican en grupo. Con palos de paleta hacen portalápices y los pintan (imitando la figura de lápices de color). Los alumnos de Creatividad planifican y se les aprueban los proyectos y los dan después a los niños de enseñanza básica. Hacen trabajos con 'fomi' (espuma), ellos (los estudiantes) viene a dar las clases en el turno contrario a su horario de estudios. En Teatro, trabajan dramatización de cuentos, narraciones salvadoreñas, fábulas de Esopo y mitología. Cuentan con una parte de los materiales, con guitarra y CD player».

Estas experiencias son elocuentes sobre la relevancia que cobra la Educación Artística en la vida de los estudiantes. Los docentes han logrado elevar la calidad de esta disciplina hacia una experiencia significativa de aprendizaje, la cual aprovechan los niños y los jóvenes. Con ello ganan nuevas perspectivas sobre su proyecto de vida y, además, el efecto positivo de su aporte a la sociedad en la que se desenvuelven. ${ }^{19}$

\section{Conclusiones}

Los resultados arrojados por el estudio La Educación Artística en la enseñanza básica en El Salvador (Valle Contreras, 2011) señalan que la asignatura de Educación Artística en los centros escolares del sector público se imparte de manera precaria, por causa de los siguientes factores (de carácter estructural) que afectan la calidad de la enseñanza e, incluso, propician el hecho de no brindar esta asignatura regularmente en algunos centros escolares:

\footnotetext{
${ }^{19}$ Para ampliar, ver: La Educación Artística en la enseñanza básica en El Salvador (Valle Contreras, 2011, p.226). Cuadro comparativo de respuestas entre las investigaciones La Educación Artística en la Enseñanza Básica en El Salvador, (Valle Contreras,2009) y Globalización, Neoliberalismo y Reforma Educativa 1994-1998, (Gregorio Lorenzo Ramírez Ayala, Norma Alicia Flores Figueroa y Mirna Elizabeth Ágreda de Iraheta, 1998).
} 
a) La baja prioridad de la Educación Artística en la sociedad salvadoreña.

b) La ausencia de programas de formación docente especializados en Educación Artística.

c) Poco acceso a recursos (humano, equipo, materiales e infraestructura) para la Educación Artística en la enseñanza básica.

d) Los paradigmas que subyacen en la base del enfoque de los docentes a la asignatura de Educación Artística en los centros escolares; lo cual los lleva a priorizar las llamadas materias básicas (Ciencias Sociales, Ciencias y Medio Ambiente, Matemáticas, Lenguaje) sobre la asignatura de Educación Artística por considerarla «menos importante» para el desempeño laboral e innecesaria para el desarrollo y la educación integral del alumnado. Por otro lado, existe el sesgo de un enfoque pseudoacademicista ${ }^{20}$ en el nivel de enseñanza básica en algunos centros escolares.

Teniendo otro objetivo diferente del academicista, la Educación Artística en el currículo nacional -en el consenso internacional se trata de educación Estética y Cultural- y tomando en cuenta que existe un vasto corpus de la teoría y práctica de la Educación Artística para el entorno escolar y como

\footnotetext{
${ }^{20}$ About en español. «Historia del academicismo». http://arte.about.com/od/Historia-delarte/fl/Historia-del-academicismo.htm Academicismo como tendencia artística se refiere a la enseñanza en las academias de arte dirigidas por un artista; se remontan al siglo XVI en Italia, en los talleres de producción y reproducción de arte donde el maestro enseñaba a los aprendices. El concepto de academias de arte se instala en Europa y para el siglo XIX existen un centenar de estas, de las cuales la que se considera modelo es la Academia de Bellas Artes de París. Este enfoque educativo, al tener como fin último la enseñanza de carácter disciplinar conducente a la profesionalización además de tener a la base el profundo dominio disciplinar del maestro, también implica una didáctica particular y propia que lo caracteriza. El academicismo del siglo XIX se caracteriza, entre otros aspectos por: ser conservador, el virtuosismo técnico, dicta el arte oficial, por el clasicismo, ser alegórico, enfatiza la idealización, es historicista, etc. En algunos centros escolares se encuentran elementos pseudo-academicistas en el contexto de la asignatura de Educación Artística. Academicismo como calificativo puede tener una connotación peyorativa por referirse a la rigidez de las academias de arte europeo del siglo XIX en contraposición al arte contemporáneo. También puede denotar a la obra que responde a las doctrinas de una academia contemporánea al artista que la creó.
} 
área de conocimiento, en El Salvador cada nueva generación de estudiantes pierde la oportunidad de que dicha forme parte del currículo nacional.

En esto resulta fundamental la adecuada formación del profesorado de educación básica y media en la rama de Educación Artística. Lamentablemente, las evidencias muestran que dicha formación no es actualizada, sólida, ni tampoco cuenta con el estudio necesario. A esto se suma que el país cuenta con pocos docentes especializados en Educación Artística, dado que hasta 2015 se abrió el primer profesorado en esta rama educativa en una universidad salvadoreña.

\section{Prioridad que ha tenido (y tiene) la educación artística en la sociedad salvadoreña}

Se concluye que la prioridad brindada por la sociedad salvadoreña a la Educación Artística en el país es baja, en lo concreto y en la práctica; en comparación a otros países de Centroamérica, Latinoamérica y del mundo. Por tal razón, en El Salvador no existen programas de Educación Superior para la formación docente especializada en Educación Artística, aunque esta es importante para garantizar el derecho humano y de la niñez que contempla el acceso a una educación integral. Asimismo, resulta escasa la oferta educativa para la formación profesional de artistas. Otro aspecto que evidencia lo anterior es la forma precaria en que se imparte la Educación Artística en los centros escolares públicos.

Todo esto se reitera en la reseña histórica sobre la Educación Artística en el currículo nacional, realizada en el estudio de 2011, y su relación con las agendas políticas, económicas y culturales desde el Período Colonial Tardío a la actualidad; la cual denota una dinámica fluctuante con relación a la importancia que la sociedad salvadoreña (y los tomadores de decisión en El Salvador ) le ha dado en la modernidad a la Educación Artística, tanto en la formación de artistas profesionales como a la Educación Artística en el currículo, al igual que al desarrollo cultural del país.

La investigación demostró un desinterés de los gobiernos en el desarrollo cultural y artístico del país, desde finales de los 70, lo cual se ha demostrado en cada currículo de enseñanza básica, como también en la decadencia 
(de forma progresiva) de la formación docente en el área de Educación Artística, desde esos años hasta el presente; así como también en la ausencia de programas de formación docente en esa área educativa.

Otro aspecto que denota lo antes mencionado es la forma limitada en que se enseña la Educación Artística en los centros escolares públicos, aunque hay excepciones.

Aspecto que en el nuevo estudio que estoy por publicar se muestra como de interés atender de parte del MINED al ser una deuda histórica impostergable para el país y ante la demanda que impone la realidad del contexto salvadoreño para que la educación artística en el currículo nacional sea una herramienta que junto a las otras áreas que lo componen, apoye procesos de prevención de la violencia que coadyuven a restituir el tejido social y la convivencia ciudadana, además de cumplir un fin en sí misma para el desarrollo humano integral del alumnado, en los términos que se explica a partir del segundo epígrafe en este artículo.

\section{Ausencia de desarrollo disciplinario ante la falta de programas docentes de especialización en Educación Artística en el país}

La investigación histórica, y las fases 1 y 2 de este estudio, corroboran que existe una ausencia de desarrollo disciplinario por falta de programas de formación docente en Educación Artística en El Salvador. Se genera un círculo vicioso que tiene como elementos concomitantes: la ausencia de plazas para docentes especializados (salvo pocas excepciones) en esta rama educativa, en los centros escolares del sector público, como en espacios de educación no formal en el sector municipal.

El otro elemento en este círculo es que, por lo anterior, no hay demanda de programas de educación superior en esta área y, en consecuencia, tampoco se genera la oferta por parte de las universidades del país. Por tanto, quienes imparten la asignatura son los maestros de grado, quienes no cuen$\tan$ con la formación especializada ni actualizada en Educación Artística, aun cuando en algunos casos estos han recibido uno o dos semestres de esa asignatura durante su formación docente o en capacitaciones. 
La tendencia comprobada es que tanto formadores de docentes, como docentes en servicio, abordan la asignatura como se hacía en occidente a finales del siglo XIX. Esto demuestra un estancamiento disciplinario por falta de formación docente especializada, que afecta directamente a la Educación Artística impartida en enseñanza básica, en los centros escolares. Al respecto, se encuentra una tendencia marcada, la cual indica que los alumnos no tienen acceso a una Educación Artística de calidad bajo parámetros contemporáneos en los centros escolares públicos, siendo uno de los factores primordiales en esta problemática la falta de sustento epistemológico de la Educación Artística en El Salvador, como consecuencia de la no profesionalización de los docentes en ese campo educativo.

\section{Poco acceso a recursos (humanos, materiales e infraestructura) para la Educación Artística en la enseñanza básica en los centros escolares del sector público visitados}

La actitud social frente a la Educación Artística en el país encuentra su contrapartida en las políticas y el presupuesto del Estado (esta investigación se cerró en diciembre de 2009). La falta de recursos financieros es una de las razones por las cuales los docentes de grado -y no docentes especialistas en Educación Artística- imparten la asignatura en los centros escolares públicos (salvo excepciones) y por lo que faltan otros recursos como materiales e infraestructura para su desarrollo. Igualmente, se da el caso que la asignatura de Educación Artística no se enseña de manera adecuada en algunos centros escolares públicos, por priorizar otras asignaturas del currículo de enseñanza básica.

\section{Paradigmas que subyacen en la base del enfoque que los docentes le dan a la asignatura de Educación Artística en los centros escolares del sector público visitados y los demás maestros y expertos entrevistados}

La Educación Artística en la enseñanza básica, en los centros escolares del sector público, se desarrolla como se impartía a finales del siglo XIX en el 
mundo occidental. Esto incluye muchos de los paradigmas educativos que en Educación Artística predominaban en esa época; por lo tanto, es necesaria la profesionalización de los docentes dedicados a esta área de la enseñanza y, de esta manera, actualizar los programas de formación docente para superar esta carencia en El Salvador.

El hecho de que en los centros escolares del país se reproduce una Educación Artística no acorde con los enfoques contemporáneos de la asignatura en el mundo, revela además que -en relación a los programas de estudio de la Educación Artística que entraron en vigencia en enero de 2009- la enseñanza en los centros escolares visitados dista de manera significativa de lo propuesto en dicha edición de los programas aún vigentes, en diciembre de 2009. De tal manera que hay una significativa distancia entre lo declarado en los programas (en los preliminares y contenidos) y lo desarrollado por los docentes en los centros escolares, debido al conjunto de aspectos planteados en este estudio como factores estructurales que obstaculizan el desarrollo de la asignatura en el sector público.

Así, para crear las condiciones con el fin de potenciar la asignatura de Educación Artística en el currículo nacional - de manera que coadyuve a incidir en la realidad nacional en coherencia con el Plan Nacional de Educación-, las experiencias estéticas (de acuerdo a Aguirre,2005; Torres Eça,2010) en la Educación Artística, como se ha perfilado en este artículo, que conllevan las prácticas artísticas (descritas por Torres Eça, 2010) deben brindar al alumnado la oportunidad de vivenciar en la asignatura de Educación Artística, en el sistema educativo nacional, procesos psicológicos y sociales de construcción de significados y de enriquecimiento cultural.

Por tal razón es imprescindible pensar la asignatura de Educación Artística desde una perspectiva técnica, tomando en cuenta los avances y consensos internacionales en Educación Artística, los nuevos discursos de las artes y el acervo nacional a partir de necesidades, intereses, problemas y riquezas del país. Para ello, se vuelve indispensable que el Ministerio de Educación cree espacios y convoque equipos multidisciplinarios de especialistas informados sobre esta visión renovada de la asignatura; validada por toda la comunidad internacional de arte, educadores y gestores en esta área, 
aglutinados en foros representativos de todas las regiones del mundo. ${ }^{21}$ Este replanteamiento necesario dará la pauta para diseñar, de manera consecuente, la formación docente en Educación Artística en el país.

La sociedad salvadoreña puede potenciar esta valiosa oportunidad, si la asignatura de Educación Artística es mediada desde un enfoque que hace posible los alcances de la misma en los términos aquí esbozados. De lo contrario, el sistema educativo cae en la práctica artística de carácter tecnicista o instrumental, cuyos alcances son limitados para apoyar procesos de desarrollo cultural con incidencia en la realidad nacional.

\section{Avances}

Existen avances en cuanto a la Educación Artística en la agenda educativa nacional, sobre todo en términos del interés del Ministerio de Educación por buscar vías que mejoren la calidad de la Educación Artística en el sistema; aspecto que todavía es una deuda histórica en el país. Este primer paso se tradujo en que el MINED desarrolló el currículo para el Profesorado en Educación Artística y convocó a las universidades del país, en 2014, para crear esa oferta educativa. Hasta la fecha, solo una universidad en el oriente del país ofrece este profesorado. En 2015, inició la formación de especialistas en Educación Artística como parte del Plan Nacional de Formación Docente.

En el área de educación, en este Plan, es necesario no reproducir un paradigma instrumentalista y tecnicista de la asignatura, en los términos explicados, para que los docentes replanteen la materia en el sistema. Al mismo tiempo, es necesario acompañar oportunamente a estos docentes con infraestructura, material y equipo; al igual que garantizar el uso de estos elementos para obtener una visión renovada de la Educación Artística., a fin de aprovechar al máximo la inversión.

Los pasos mencionados demuestran que hay interés en esta instancia del Gobierno por mejorar la calidad de la Educación Artística en el sistema educativo nacional, en particular en el sector público. Sin embargo,

\footnotetext{
${ }^{21}$ Para ampliar sobre estas dinámicas, ver: Internacional Society for Education Through Art http:// insea.org/;World Alliance for Arts Education y http://waae.edcp.educ.ubc.ca/
} 
la problemática de la educación artística en el país -siendo aún una deuda histórica y de carácter estructural- demanda alineamiento de varias instancias y una visión estratégica para concretar la ejecución de una planificación con metas a corto, mediano y largo plazo, las cuales trasciendan los períodos presidenciales.

\section{Estrategia fundacional hacia una educación artística de calidad $^{22}$}

Tantas décadas de no atender el área artística en el país y, en consecuencia, en el currículo nacional -como reflejo de lo primero-, ha generado desafios de carácter estructural en la educación artística dentro del sistema educativo, lo cual demanda pensar en una Estrategia Nacional para el Fomento de la Educación Artística desde el abordaje integral, sistémico y estratégico de la problemática para llegar al escenario deseado.

Por ahora $-y$ ante la escasa existencia de docentes especialistas en educación artística y también por la mínima oferta formativa en esta áreacabe planificar de manera estratégica para que la oferta docente especializada crezca sustancialmente, a fin de que a largo plazo sea posible que cada centro escolar, en el sistema público, tenga en su personal (por lo menos) un docente especialista en Educación Artística a tiempo completo; que cuente con la infraestructura, materiales y equipo para gestionar la asignatura en coordinación con el maestro responsable de grado en educación básica. Esto debe asegurar la calidad de la formación docente; así como también crear condiciones para recuperar la asignatura de Educación Artística (Estética y cultural) en educación media.

Hay que imaginarlo, formularlo, planificarlo y trabajar hacia la dirección del escenario deseado. Para lograrlo, es fundamental el abordaje desde una perspectiva estratégica. Ha tomado muchas décadas en el país para

\footnotetext{
${ }^{22}$ Aquí se incluye una recomendación general, con relación a las cuatro causas estructurales y su estado, al momento de cerrar la edición del estudio publicado en 2011. En esa publicación se agrega el cuadro Congruencia del proceso de investigación (p.230, en el cual se hacen recomendaciones específicas para cada causa estructural planteada con relación al desarrollo de la educación artística, en el currículo nacional.
} 
que la Educación Artística se valorice en las instancias gubernamentales, por ello es importante enfocarse en la calidad de los procesos que se dan, como los iniciados por el MINED.

En este sentido, es fundamental dar los tiempos, recursos y condiciones necesarias a estos procesos (haciendo énfasis en garantizar la calidad de la formación docente) para adquirir las competencias que los lleve profesorado a generar experiencias significativas de aprendizaje en Educación Artística para el alumnado. Para que esto suceda es fundamental que los formadores de formadores dominen un área artística y otra complementaria, a la vez que cuenten con una formación actualizada y profunda de la cultura nacional, de los avances internacionales en Educación Artística y de los nuevos discursos artísticos, de cara a las necesidades del contexto salvadoreño para evitar que en los centros escolares se reproduzcan las fallas aquí expuestas.

Esto es la base para que la Educación Artística en el país se encamine hacia los nuevos paradigmas que lleven a potenciar esta asignatura como una oportunidad para el desarrollo del alumnado en el aspecto humano, cultural y ciudadano.

Para el abordaje estratégico de la Educación Artística, en el sistema educativo nacional, también es necesario el alineamiento del Ministerio de Educación (MINED), la Secretaría de Cultura de la Presidencia (SECULTURA) y la comisión de creación del Instituto Superior de Artes (ISAR), y a las otras Instituciones de Educación Superior, igualmente es preciso trabajar con los consultores independientes -especialistas en Educación Artística y Educación Biocéntrica- y así garantizar cambios sustanciales en el desarrollo de la Educación Artística con calidad en los centros escolares y espacios municipales del país.

Todo este esfuerzo debería orientarse hacia la ejecución de una Estrategia Nacional para el fomento de la Educación Artística, cuyo objetivo sea generar cambios actitudinales de la población (incluyendo funcionarios y tomadores de decisión) hacia las artes y la cultura salvadoreña, al empoderamiento estético y cultural de los salvadoreños. En esto juegan un papel fundamental diversos procesos educativos, incluyendo la formación docente, que se traduzcan en el interés de todos los sectores en tutelar, promover y difundir las artes y la cultura local. 
En definitiva, es urgente crear las condiciones para potenciar la oportunidad que brinda la Educación Artística en el currículo nacional y así construir una sociedad que se conciba como protagonista de la dinámica de creación, re-creación y consumo artístico cultural local, como fundamento para el desarrollo humano integral y sostenible para reconstruir el tejido social hacia una cultura de paz y para el fomento de la economía cultural de El Salvador. 


\section{REFERENCIAS BIBLIOGRÁFICAS}

Aguirre, I. Teorías y prácticas en educación artística: ideas para una revisión pragmatista de la experiencia estética. Barcelona: Octaedro, S.L., 2005.

Barbosa,A. M. Revista de Educação da APEOESP. Obtenido de http://www. apeoesp.org.br/: www.apeoesp.org.br/d/sistema/publicacoes/.../revista-de-arte.pdf, 2010.

Eça,T.T.A Educaçao artística e as prioridades educativas do inicio do seculo XXI. Revista Ibero Americana de Educaçao. N. ${ }^{\circ} 52,127-146$. Recuperado de rieoei.org/rie52a07.pdf, 2010. Consultado el 18 de agosto de 2016.

Flórez Ochoa, R. T. Investigación educativa y pedagógica. Bogotá: McGraw Hill Interamericana, S.A.1997.

Lasso, S. About en Español. Obtenido de http://arte.about.com/od/Historiadel-arte/fl/Historia-del-academicismo.htm. Consultado el 18 de agosto de 2016.

Medina, M., López Cerezo, A., \& Sánchez Ron [Eds], M. Ciencia Tecnología Sociedad y Cultura: Ciencia y tecnología como sistemas culturales. Madrid: Biblioteca Nueva, S.L., 2001.

Navas, V. La Investigación Social. San Salvador: Centro de Investigaciones en Ciencias y Humanidades (CICH-UJMD), Universidad Dr. José Matías Delgado, 2012.

Nusbaum, M. El duro discurso. Discurso al recibir el doctorado honoris causa de parte de la Universidad de Antioquia. 10 de Diciembre de 2015. Obtenido de http://www.elheraldo.com: http://www.elheraldo.co/educacion/elduro-discurso-de-martha-nussbaum-sobre-el-futuro-de-la-educacionmundial-233416. Consultado el 18 de agosto de 2016.

Sampieri, R. H., Fernández Collado, C., \& Batista Lucio, P. Metodología de la Investigación. México: Mc Graw Hill,1997.

Valle, M. E. La Educación Artística en la Enseñanza Básica en El Salvador. Antiguo Cuscatlán: Centro de Investigaciones en Ciencia y Humanidades (CICH), 2011. 